Article

\title{
Minimization of the Energy Consumption in Industrial Robots through Regenerative Drives and Optimally Designed Compliant Elements
}

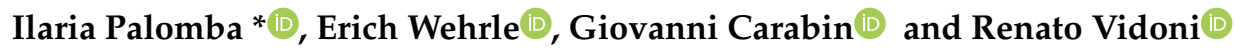 \\ Faculty of Science and Technology, Free University of Bozen-Bolzano, Piazza Università 5, 39100 Bolzano, Italy; \\ erich.wehrle@unibz.it (E.W.); giovanni.carabin@unibz.it (G.C.); renato.vidoni@unibz.it (R.V.) \\ * Correspondence: ilaria.palomba@unibz.it; Tel.: +39-0471-017-757
}

Received: 8 October 2020; Accepted: 21 October 2020; Published: 24 October 2020

check for updates

\begin{abstract}
This paper describes a method for reducing the energy consumption of industrial robots and electrically actuated mechanisms performing cyclic tasks. The energy required by the system is reduced by outfitting it with additional devices able to store and recuperate energy, namely, compliant elements coupled in parallel with axles and regenerative motor drives. Starting from the electromechanical model of the modified system moving following a predefined periodic path, the relationship between the electrical energy and the stiffness and preload of the compliant elements is analyzed. The conditions for the compliant elements to be optimal are analytically derived. It is demonstrated that under these conditions the compliant elements are always beneficial for reducing the energy consumption. The effectiveness of the design method is verified by applying it to two test cases: a five-bar mechanism and a SCARA robot. The numerical validations show that the system energy consumption can be reduced up to the $77.8 \%$ while performing a high-speed, standard, not-optimized trajectory.
\end{abstract}

Keywords: energy efficiency; industrial robot; regenerative drives; compliant elements; springs; cyclic tasks

\section{Introduction}

The ever-growing awareness of the negative consequences on the environment of the energy needs of manufacturing systems has led to the study and development of methodologies to reduce energy consumption. Industrial robots make up a significant part on the total electrical energy consumed in the manufacturing sector [1]. Studies to reduce the energy consumption of these systems have been recently reviewed by Carabin et al. [2]. Two main categories have been identified: software and hardware solutions.

The software approaches rely mainly on the optimization of system motion planning and, therefore, require a modification of just the software that handles the robotic operations. Improvements in energy efficiency can be achieved by optimizing the location of the task with respect to the base of the manipulator [3] or by exploiting the system intrinsic or functional kinematic redundancy [4-6]. Other approaches are based on the trajectory optimization toward minimizing the energy consumption of point-to-point [7], multipoint [8] trajectories, and maximizing the energy sharing in multi-axis robots provided with a common Direct Current (DC) bus $[9,10]$.

The energy efficiency enhancement by means of hardware solutions is obtained by introducing physical modifications to the system. An example of hardware solution is the lightweight design of the system and its components [11] by means of, e.g., the use of novel materials [12]. The energy efficiency of an industrial robot can be improved by replacing its traditional drives with regenerative 
drives [13-16]. These devices convert the braking energy of the system into regenerative electric energy that is returned to the system when required, instead of dissipating it as heat. Another way to store energy for transferring it to the system consists in providing the system with energy-storing devices such as flywheels [17] or compliant elements as, e.g., springs.

The adoption of springs is by far most used in robotic systems with respect to flywheels. They are typically installed at robot joints in a serial or parallel connection [18], see Figure 1. The serial arrangement generally requires to substitute stiff actuators with compliant ones. Such a configuration is typically adopted to reduce force peaks due to impacts as for example in human-robot interaction. Conversely, the parallel configuration does not require the substitution of the original actuators, resulting in an easier and cheaper installation.

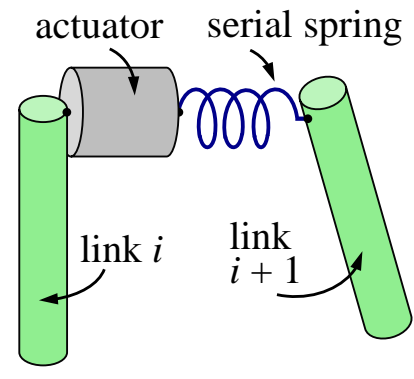

(a)

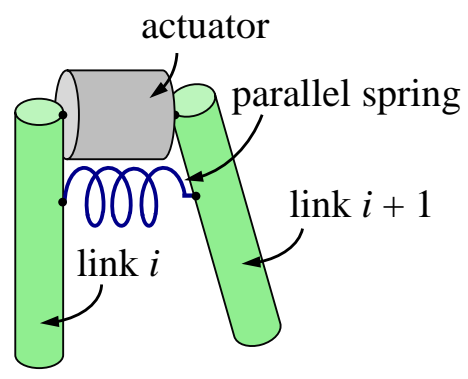

(b)

Figure 1. Serial (a) and parallel (b) spring configuration as shown in Scalera et al. [19].

The addition of springs to robotic systems for reducing the actuator effort was first adopted for gravity balancing $[20,21]$. Gravity balanced robots are more energy efficient than non-equilibrated ones as they do not need actuator effort to stay in equilibrium at any configuration.

Methods that use springs to improve the efficiency of robotic systems in repetitive operations are based on the concept of the natural motion [19]. The basic idea of these methods is to shape by means of a proper tuning of the spring stiffness the free vibration response of the robot so that it matches with the repetitive tasks that the robot has to perform. An iterative procedure to exploit the system free response for performing pick-and-place tasks between two given points in a prescribed amount of time is presented in $[22,23]$. A multibody simulator is used to simulate the free response of the system for the given initial position and for guess values of the springs mounted in parallel with the actuators. Simulations are stopped when the optimal spring values are found, i.e., when after the desired time the position of the system is close enough to the final given position.

In [24], adjustable compliant actuators mounted in series with the joints are used to exploit the natural dynamics of serial robots. The actuator stiffnesses are tuned by imposing the desired natural frequencies in the decoupled modal model of the system obtained through linearization of the dynamic model. A control scheme based on a feedback linearization method is then used to generate smooth reference link positions that excite the mode corresponding to the desired periodic motion that is performed employing a small amount of energy.

Linearization and modal analysis techniques are used also in $[25,26]$ to minimize the consumed mechanical energy of actively controlled multibody systems moving between two given points. In particular, the optimal spring design, the optimal trajectory, and the operating time are determined using the optimal control theory. A concurrent optimization of the springs and the trajectory is performed also in $[27,28]$ where both the trajectory and the nonlinear spring profile are parameterized by means of polynomial functions whose coefficients are determined to minimize actuation torques.

All methods provide efficient ways to tune the elastic elements added to the system for minimizing the energy consumption, but they also impose constraints on the kind of path that the robot must follow, i.e., they do not allow to use an arbitrary trajectory for the operation. This can be a drawback 
in several scenarios, e.g., when an obstacle avoidance planning is necessary, if the robot controller allows only for the implementation of standard motion primitives, if an optimal motion planning with a different objective function is to be used, such as minimum execution time [29] or smoothness of the motion profile [30].

Few works in the literature deal with the optimal design of springs when the system moves following an arbitrary, given trajectory. Additionally, all these works have been developed for a specific type of system. In [31], a constrained optimization problem is formulated to reduce the peak torque requirement of a one-dof rotating link by finding the stiffness value and placement of a linear spring to be added in parallel with the joint actuator. In [32], springs in parallel with the actuators are used to minimize the mechanical energy required by the motors of a four-dof parallel robot performing predefined pick-and-place trajectories. In [33], an analytical method has been developed with reference to a one-dof rotating beam provided with a regenerative drive. The method allows to find the optimal design of the spring balancing the system that minimizes the electric energy of rest-to-rest motions. The authors of the present work in [34] achieved energy consumption minimization retrofitting a Delta-2 robot with both regenerative drives recovering braking energy and springs, properly tuned for a chosen trajectory by means of numerical optimization, storing elastic energy.

As promising results have been obtained (e.g., energy reduction up to $70 \%$ ), this paper further develops and generalizes such an approach for robotic systems performing cyclic tasks. In particular, the electromechanical model of the system provided with regenerative drives and linear springs mounted in parallel with the actuators is described. It is demonstrated analytically that the addition of the springs is always beneficial for reducing the energy consumption of the system if they are properly designed in terms of stiffness and preload. The optimal values for the spring parameters are analytically determined through a bounded optimization problem whose objective function is the reduction of the electrical energy consumption. No constraints are set on the system trajectory, so the energy efficiency can be improved for any arbitrary trajectory (e.g., the current one or an energy-optimal motion profile to further boost the energy savings). Numerical results of the two test cases, a five-bar mechanism and a SCARA robot, show that the consumed energy can be significantly reduced. The paper is organized as follows. Section 2 reports the electromechanical model of robots provided with regenerative drives. Section 3 describes the model of the system with linear elastic elements mounted in parallel with the actuator and the optimal design of these components. Section 4 shows the results of the numerical simulations. Finally, Section 5 draws the conclusions.

\section{Electromechanical Model}

Let us consider a fully-actuated $n$-dof robot with rigid links and either closed or open kinematic chain. Let $t$ be the time variable and $\mathbf{q}=\mathbf{q}(t) \in \mathbb{R}^{n}$ be the vector of the generalized coordinates of the actuated joints. The torques $\boldsymbol{\tau}=\boldsymbol{\tau}(t) \in \mathbb{R}^{n}$ that the actuators must exert to move the system from the initial time $t_{0}$ to the final one $t_{f}$ along a given trajectory in the joint space $\left(\mathbf{q}(t)\right.$ with $\left.t \in\left[t_{0} ; t_{f}\right]\right)$ can be computed from the system equations of motion as follows:

$$
\boldsymbol{\tau}=\mathbf{I K}_{r}^{-1} \ddot{\mathbf{q}}+\mathbf{K}_{r}\left(\mathbf{M}(\mathbf{q}) \ddot{\mathbf{q}}+\mathbf{f}_{c}(\mathbf{q}, \dot{\mathbf{q}})+\mathbf{F}_{v} \dot{\mathbf{q}}+\mathbf{f}_{s}(\mathbf{q}, \dot{\mathbf{q}})+\mathbf{f}_{g}(\mathbf{q})+\mathbf{f}_{e}(\mathbf{q})\right)
$$

where

- $\quad \dot{\mathbf{q}}=\dot{\mathbf{q}}(t) \in \mathbb{R}^{n}$ and $\ddot{\mathbf{q}}=\ddot{\mathbf{q}}(t) \in \mathbb{R}^{n}$ denote the first and second time derivative of vector $\mathbf{q}$, i.e., the vectors of the actuated joint velocities and accelerations, respectively;

- $\mathbf{I} \in \mathbb{R}^{n \times n}$ is the diagonal matrix of the motor inertia;

- $\quad \mathbf{K}_{r} \in \mathbb{R}^{n \times n}$ is the diagonal matrix of the gear transmission ratios;

- $\mathbf{M}(\mathbf{q}) \in \mathbb{R}^{n \times n}$ is the mass matrix;

- $\quad \mathbf{f}_{c}(\mathbf{q}, \dot{\mathbf{q}}) \in \mathbb{R}^{n}$ is the torque vector due to centrifugal and Coriolis effects;

- $\quad \mathbf{F}_{v} \in \mathbb{R}^{n \times n}$ is the diagonal matrix of viscous friction coefficients; 
- $\mathbf{f}_{s}(\mathbf{q}, \dot{\mathbf{q}}) \in \mathbb{R}^{n}$ is the vector of the static friction torques, that may be represented by means of the Coulomb friction model. In this case $\mathbf{f}_{s}=\mathbf{F}_{s} \operatorname{sign}(\dot{\mathbf{q}})$, where $\mathbf{F}_{s} \in \mathbb{R}^{n \times n}$ is a diagonal matrix of the static friction coefficients;

- $\quad \mathbf{f}_{g}(\mathbf{q}) \in \mathbb{R}^{n}$ is the torque vector due to the effects of gravity;

- $\quad \mathbf{f}_{e}(\mathbf{q}) \in \mathbb{R}^{n}$ is the torque vector due to the interaction of the end-effector with the environment.

The robotic system considered is actuated by means of DC or permanent magnet synchronous (PMS) motors. The vector of the actuation torques in Equation (1) is related to the armature current vector, $\boldsymbol{i} \in \mathbb{R}^{n}$, of the motors by means of the diagonal matrix of the motor torque constants $\mathbf{K}_{t} \in \mathbb{R}^{n \times n}$ :

$$
\boldsymbol{i}(t)=\mathbf{K}_{t}^{-1} \boldsymbol{\tau}(t)
$$

The supply voltage of the motor drivers $e \in \mathbb{R}^{n}$ is given by:

$$
\boldsymbol{e}(t)=\mathbf{R} \boldsymbol{i}(t)+\mathbf{K}_{e} \mathbf{K}_{r}^{-1} \dot{\mathbf{q}}(t)
$$

where $\mathbf{R} \in \mathbb{R}^{n \times n}$ and $\mathbf{K}_{e} \in \mathbb{R}^{n \times n}$ are diagonal matrices of the resistances of the motor windings and the velocity constants of the motors, respectively. $\mathbf{K}_{r}^{-1} \dot{\mathbf{q}}(t)$ is the motor speed. In Equation (3), the inductive terms have not been considered since the energy dissipated in the inductances in rest-to-rest motion is equal to zero [35]. The instantaneous input power, $P$, to the motor drivers is given by the product of Equations (2) and (3), that it is equivalent to the sum of the instantaneous input power to each motor driver, $P_{j}$ :

$$
P(t)=\boldsymbol{e}(t)^{\mathrm{T}} \boldsymbol{\eta}^{-1} \boldsymbol{i}(t)=\sum_{j=1}^{n} P_{j}(t)=\sum_{j=1}^{n} \frac{e_{j}(t) i_{j}(t)}{\boldsymbol{\eta}_{j}}
$$

where $\boldsymbol{\eta} \in \mathbb{R}^{n \times n}$ is the diagonal matrix of the efficiency of each driver, $\boldsymbol{\eta}_{j}, j=1, \ldots, n$. According to the passive sign convention, $P_{j}$ is positive and here denoted as $P_{c, j}$ if the power flows from the network to the system, i.e., if the system consumes power:

$$
P_{c, j}=\left\{\begin{aligned}
P_{j} & \text { if } P_{j} \geq 0 \\
0 & \text { if } P_{j}<0
\end{aligned}\right.
$$

Conversely, $P_{j}$ is negative if the electric power flows out of the actuator. In general, such power is passed through resistors and dissipated as heat, unless regenerative devices are used. In this case, the power flowing out of the system is stored in capacitors and reused as regenerated power $P_{r, j}$ :

$$
P_{r, j}=\left\{\begin{aligned}
0 & \text { if } P_{j} \geq 0 \\
-P_{j} & \text { if } P_{j}<0
\end{aligned}\right.
$$

The energy consumption of a robot provided with traditional, non-regenerative drivers is given by the sum of time integral of the power consumed by each motor $P_{c, j}$ :

$$
E=\int_{t_{0}}^{t_{f}} P_{c}(t) d t=\sum_{j=1}^{n} \int_{t_{0}}^{t_{f}} P_{c, j}(t) d t
$$

If regenerative drivers are used, as it is assumed in this work, the electrical energy consumed by the robot is smaller, as the energy necessary to move the system is taken not only from the network but also from the capacitors that store the braking energy. The net energy consumption is given by the sum of the time integral of both the consumed power $P_{c, j}$ and the regenerated one $P_{r, j}$ of each motor, that corresponds to the time integration of Equation (4): 


$$
\begin{aligned}
E & =\int_{t_{0}}^{t_{f}} P_{c}(t) d t-\int_{t_{0}}^{t_{f}} P_{r}(t) d t=\int_{t_{0}}^{t_{f}} P(t) d t \\
& =\sum_{j=1}^{n} E_{j}=\sum_{j=1}^{n} \int_{t_{0}}^{t_{f}} P_{j}(t) d t=\sum_{j=1}^{n} \int_{t_{0}}^{t_{f}} \frac{e_{j}(t) i_{j}(t)}{\eta_{j}} d t
\end{aligned}
$$

\section{Optimal Design of Compliant Elements}

The adoption of the regenerative drives leads to a reduction of the energy consumption, as is evident from the comparison of Equations (7) and (8). It is possible to further reduce the energy consumption by providing the robot with compliant elements, i.e., springs. Compliant elements store elastic energy and release it as kinetic energy, which then in addition to actuation moves the system. Different ways exist to add compliant elements to a system as described in [19]. In this study, parallel springs are added to the actuated joints of the robot. The moment (torque) exerted by the springs is:

$$
\boldsymbol{\tau}_{e}(t)=\boldsymbol{K}(\mathbf{q}(t)-\boldsymbol{\theta})
$$

where $\boldsymbol{K}=\operatorname{diag}\left(k_{1}, \ldots, k_{n}\right)$ is the elastic stiffness matrix of the added springs and $\boldsymbol{\theta}$ is the vector of the spring mounting positions. The torque of the springs must be included in the equations of motion for the system (Equation (1)):

$$
\begin{aligned}
\tilde{\boldsymbol{\tau}}(t)= & \underbrace{\mathbf{I} \mathbf{r}_{r}^{-1} \ddot{\mathbf{q}}+\mathbf{K}_{r}\left(\mathbf{M}(\mathbf{q}) \ddot{\mathbf{q}}+\mathbf{f}_{c}(\mathbf{q}, \dot{\mathbf{q}})+\mathbf{F}_{v} \dot{\mathbf{q}}+\mathbf{f}_{s}(\mathbf{q}, \dot{\mathbf{q}})+\mathbf{f}_{g}(\mathbf{q})+\mathbf{f}_{e}(\mathbf{q})\right)}_{\boldsymbol{\tau}(t)}+\mathbf{K}_{r} \underbrace{\mathbf{K}(\mathbf{q}-\boldsymbol{\theta})}_{\boldsymbol{\tau}_{e}(t)} \\
= & \left\{\begin{array}{c}
\boldsymbol{\tau}_{1} \\
\vdots \\
\boldsymbol{\tau}_{j} \\
\vdots \\
\boldsymbol{\tau}_{n}
\end{array}\right\}+\left\{\begin{array}{c}
\mathrm{K}_{r, 1} k_{1}\left(q_{1}-\boldsymbol{\theta}_{1}\right) \\
\vdots \\
\mathrm{K}_{r, j} k_{j}\left(q_{j}-\boldsymbol{\theta}_{1}\right) \\
\vdots \\
\mathrm{K}_{r, n} k_{n}\left(q_{n}-\boldsymbol{\theta}_{n}\right)
\end{array}\right\}
\end{aligned}
$$

where $\widetilde{\tau}$ is the torque that includes both the contributions from the actuators as well as the added springs. As the trajectory is given, all the terms in Equation (10) are known with the exception of the spring stiffnesses $k_{j}$ and mounting positions $\boldsymbol{\theta}_{j}(j=1, \ldots, n)$. These values are to be found by minimizing the energy consumption of the system E. By substituting Equations (2), (3), and (8) into Equation (10), the following expression is obtained for the system energy consumption:

$$
\begin{aligned}
E & =\sum_{j=1}^{n} \int_{t_{0}}^{t_{f}} P_{j}(t) d t \\
& =\sum_{j=1}^{n} \underbrace{\int_{t_{0}}^{t_{f}}\left(\frac{\mathrm{R}_{j}}{\boldsymbol{\eta}_{j} \mathrm{~K}_{t, j}^{2}}\left(\boldsymbol{\tau}_{j}(t)+\mathrm{K}_{r, j} k_{j}\left(q_{j}(t)-\boldsymbol{\theta}_{j}\right)\right)^{2}+\frac{\mathrm{K}_{e, j}}{\boldsymbol{\eta}_{j} \mathrm{~K}_{t, j} \mathrm{~K}_{r, j}}\left(\boldsymbol{\tau}_{j}(t)+\mathrm{K}_{r, j} k_{j}\left(q_{j}(t)-\boldsymbol{\theta}_{j}\right)\right) \dot{q}_{j}(t)\right) d t}_{E_{j}}
\end{aligned}
$$

By analyzing Equation (11), it results that the energy consumption of each motor, $E_{j}$, depends only on the parameters, i.e., spring stiffness and mounting position, of the compliant element added to it. Therefore, the spring parameters can be determined independently for each axis:

$$
\min \left\{E\left(\boldsymbol{\theta}_{j}, k_{j}\right)\right\}=\min \left\{\sum_{j=1}^{n} E_{j}\left(\boldsymbol{\theta}_{j}, k_{j}\right)\right\}=\sum_{j=1}^{n} \min \left\{E_{j}\left(\boldsymbol{\theta}_{j}, k_{j}\right)\right\}
$$

The optimal design of the springs is determined by solving $n$ sub optimizations. For simplicity of representation we are discussing the case in which a spring is added in parallel with all the actuated system joints. However, a reduction of the energy consumption can be obtained also adopting a 
small number of springs $n_{s}$, i.e., $0<n_{s} \leq n$, as shown in Section 4.2. In this case, the number of optimizations to be solved is $n_{s}$.

By solving the integral in Equation (11), the energy consumption of motor $j$ can be more explicitly written as function of the spring parameters:

$$
E_{j}\left(\boldsymbol{\theta}_{j}, k_{j}\right)=E_{w s_{j}}+a_{j} \mathrm{~K}_{r, j} I_{q_{j}} k_{j}^{2}-2 a_{j} \mathrm{~K}_{r, j} I_{q} k_{j}^{2} \boldsymbol{\theta}_{j}+a_{j} \mathrm{~K}_{r, j} T k_{j}^{2} \boldsymbol{\theta}_{j}^{2}+2 a_{j} I_{\tau q_{j}} k_{j}-2 a_{j} I_{\tau_{j}} k_{j} \boldsymbol{\theta}_{j}
$$

where

$$
\begin{aligned}
T & =t_{f}-t_{0} \\
a_{j} & =\frac{\mathrm{R}_{j} \mathrm{~K}_{r, j}}{\eta_{j} \mathrm{~K}_{t, j}^{2}} \\
I_{q_{j}} & =\int_{t_{0}}^{t_{f}} q_{j}(t) d t \\
I_{q_{j}^{2}} & =\int_{t_{0}}^{t_{f}} q_{j}^{2}(t) d t \\
I_{\tau_{j}} & =\int_{t_{0}}^{t_{f}} \boldsymbol{\tau}_{j}(t) d t \\
I_{\tau q_{j}} & =\int_{t_{0}}^{t_{f}} \boldsymbol{\tau}_{j}(t) q_{j}(t) d t \\
E_{w s_{j}} & =\frac{\mathrm{R}_{j} \mathrm{~K}_{r, j} \int_{t_{0}}^{t_{f}} \boldsymbol{\tau}_{j}^{2}(t) d t+\mathrm{K}_{e, j} \mathrm{~K}_{t, j} \int_{t_{0}}^{t_{f}} \boldsymbol{\tau}_{j}(t) \dot{q}_{j}(t) d t}{\eta_{j} \mathrm{~K}_{t, j}^{2} \mathrm{~K}_{r, j}}
\end{aligned}
$$

The term $E_{w s_{j}}$ corresponds to the energy consumption of motor $j$ without springs. Equation (13) considers that the system performs a cyclic task, i.e., the start and the end positions coincide $q\left(t_{0}\right)=$ $q\left(t_{f}\right)$. Therefore, the following terms become zero:

$$
\begin{aligned}
\frac{\mathrm{K}_{e, j}}{\eta_{j} \mathrm{~K}_{t, j}} k_{j} \int_{t_{0}}^{t_{f}} \dot{q}_{j}(t) q_{j}(t) d t & =\frac{\mathrm{K}_{e, j}}{2 \boldsymbol{\eta}_{j} \mathrm{~K}_{t, j}} k_{j}\left(q_{j}^{2}\left(t_{f}\right)-q_{j}^{2}\left(t_{0}\right)\right)=0 \\
\frac{\mathrm{K}_{e, j}}{\boldsymbol{\eta}_{j} \mathrm{~K}_{t, j}} k_{j} \boldsymbol{\theta}_{j} \int_{t_{0}}^{t_{f}} \dot{q}_{j}(t) d t & =\frac{\mathrm{K}_{e, j}}{\boldsymbol{\eta}_{j} \mathrm{~K}_{t, j}} k_{j} \boldsymbol{\theta}_{j}\left(q_{j}\left(t_{f}\right)-q_{j}\left(t_{0}\right)\right)=0
\end{aligned}
$$

For feasibility reasons, the spring stiffnesses must be positive, and this is considered by formulating the problem as a constrained optimization problem with lower bounds $k_{j}^{L}>0$ for the stiffnesses:

$$
\begin{aligned}
& \min \left\{f_{j}\left(\mathbf{x}_{j}\right)\right\} \\
\text { subject to } & g_{j}\left(\mathbf{x}_{j}\right) \leq 0 \\
\text { where } & f_{j}=E_{j} \\
& \mathbf{x}_{j}=\left[\begin{array}{ll}
\boldsymbol{\theta}_{j} & k_{j}
\end{array}\right]^{\mathrm{T}} \\
& g_{j}=k_{j}^{L}-k_{j}
\end{aligned}
$$

In order to solve the constrained optimization problem formulated in Equations (23)-(27), the Lagrangian function is used:

$$
\begin{aligned}
\mathcal{L}\left(\mathbf{x}_{j}, \mu_{j}\right) & =f\left(\mathbf{x}_{j}\right)+\mu_{j} g_{j} \\
& =E_{w s_{j}}+a_{j} \mathrm{~K}_{r, j} I_{q_{j}^{2}} k_{j}^{2}-2 a_{j} \mathrm{~K}_{r, j} I_{q} k_{j}^{2} \boldsymbol{\theta}_{j}+a_{j} \mathrm{~K}_{r, j} T k_{j}^{2} \boldsymbol{\theta}_{j}^{2}+2 a_{j} I_{\tau q_{j}} k_{j}-2 a_{j} I_{\tau_{j}} k_{j} \boldsymbol{\theta}_{j}+\mu_{j}\left(k_{j}^{L}-k_{j}\right)
\end{aligned}
$$


where $\mu_{j}$ is the Lagrange multiplier. A design $\mathbf{x}_{j}^{*}$ is a solution if and only if the Karush-Kuhn-Tucker (KKT) conditions [36,37] are satisfied:

1. Stationary condition:

$$
\nabla_{\mathbf{x}_{j}} \mathcal{L}\left(\mathbf{x}_{j}^{*}, \mu_{j}^{*}\right)=\mathbf{0}
$$

2. Complementary slackness:

$$
\mu_{j}^{*} g\left(\mathbf{x}_{j}\right)=0
$$

3. Primal feasibility:

$$
g\left(\mathbf{x}_{j}\right) \leq 0
$$

4. Dual feasibility:

$$
\mu_{j}^{*} \geq 0
$$

Additionally, the solution $\mathbf{x}_{j}^{*}, \mu_{j}^{*}$ is a constrained minimum, if the second-order condition holds:

5. Positive definite matrix:

$$
\begin{gathered}
\mathbf{y}^{\mathrm{T}} \nabla_{\mathbf{x}_{j} \mathbf{x}_{j}}^{2} \mathcal{L}\left(\mathbf{x}_{j}^{*}, \mu_{j}^{*}\right) \mathbf{y}>0 \\
\text { with }: \mathbf{y} \neq \mathbf{0} \\
\nabla_{\mathbf{x}_{j}} g\left(\mathbf{x}_{j}^{*}\right)^{\mathrm{T}} \mathbf{y}=\mathbf{0}
\end{gathered}
$$

Two possible feasible solution types exist for such a problem: points inside the boundary $g\left(\mathbf{x}_{j}\right)<0$ (inactive constraint) and points on the boundary $g\left(\mathbf{x}_{j}\right)=0$ (active constraint). Both described in the next subsections specifically to our design case. It should be noted that a further stationary point (specifically a saddle point) exists at $k_{j}=0$, i.e., the case of no added compliance, at which corresponds an energy consumption equal to $E_{w s_{j}}$. This point will not be considered as possible solution of the problem being in the infeasible domain. However, it will be considered as benchmark case to demonstrate that the addition of the springs is beneficial for reducing the energy consumption, i.e., $E_{j}<E_{w s_{j}}$.

\subsection{Inactive Constraint $\left(\mu^{*}=0\right)$}

When the design for minimum energy consumption lies within the feasible design set, the constraint is inactive, i.e., $g\left(\mathbf{x}_{j}\right)<0$. In this case, the stationary point of the Lagrangian in Equation (28) takes the following form:

$$
\begin{aligned}
\boldsymbol{\theta}_{j}^{*(1)} & =\frac{I_{\boldsymbol{\tau}_{j}} I_{q_{j}^{2}}-I_{\tau q_{j}} I_{q_{j}}}{I_{\tau_{j}} I_{q_{j}}-T I_{\tau q_{j}}} \\
k_{j}^{*(1)} & =\frac{I_{\tau_{j}} I_{q_{j}}-T I_{\tau q_{j}}}{\mathrm{~K}_{r, j}\left(T I_{q_{j}^{2}}-I_{q_{j}}^{2}\right)} \\
\mu^{*(1)} & =0
\end{aligned}
$$

The stationary point found is a minimum point if the condition 5 of Equation (33) is verified, i.e., if the Hessian matrix $\mathbf{H}$ of the Lagrangian function with respect to the design variables is positive definite.

$$
\mathbf{H}=\nabla_{\mathbf{x}_{j} \mathbf{x}_{j}}^{2} \mathcal{L}\left(\mathbf{x}_{j}^{*(1)}, \mu_{j}^{*(1)}\right)=\left[\begin{array}{ll}
h_{11} & h_{12} \\
h_{21} & h_{22}
\end{array}\right]
$$


where

$$
\begin{aligned}
& h_{11}=\frac{2 a_{j} T}{\mathrm{~K}_{r, j}}\left(\frac{I_{\tau_{j}} I_{q_{j}}-T I_{\tau q_{j}}}{T I_{q_{j}^{2}}-I_{q_{j}}^{2}}\right)^{2} \\
& h_{12}=h_{21}=2 a_{j} I_{\tau_{j}} \\
& h_{22}=\frac{2 a_{j} \mathrm{~K}_{r, j}\left(T I_{q_{j}^{2}}-I_{q_{j}}^{2}\right)\left(I_{\tau_{j}}^{2} I_{q_{j}^{2}}-2 I_{\tau_{j}} I_{q_{j}} I_{\tau q_{j}}+T I_{\tau q_{j}}^{2}\right)}{\left(I_{\tau_{j}} I_{q_{j}}-T I_{\tau q_{j}}\right)^{2}}
\end{aligned}
$$

The Hessian matrix $\mathbf{H}$ is positive definite if the determinants of all the upper-left submatrices are positive, i.e., if the following conditions hold:

$$
\begin{aligned}
\text { 1st condition: } \quad \operatorname{det}\left(h_{11}\right)=\frac{2 a_{j} T}{\mathrm{~K}_{r, j}}\left(\frac{I_{\tau_{j}} I_{q_{j}}-T I_{\tau q_{j}}}{T I_{q_{j}^{2}}-I_{q_{j}}^{2}}\right)^{2}>0 \\
\text { 2nd condition: } \quad \operatorname{det}(\mathbf{H})=\frac{4 a_{j}^{2}\left(I_{\tau_{j}} I_{q_{j}}-T I_{\tau q_{j}}\right)^{2}}{T I_{q_{j}^{2}}-I_{q_{j}}^{2}}>0
\end{aligned}
$$

The condition in Equation (41) is always satisfied, as $a_{j}, T$, and $\mathrm{K}_{r, j}$ are positive while the other term of the product is squared. The condition in Equation (42) is satisfied if the denominator is positive, i.e., if:

$$
I_{q_{j}}^{2}<T I_{q_{j}^{2}}
$$

In order to demonstrate that this condition is satisfied, Jensen's inequality is applied.

Theorem 1. Let $f$ be an integrable function defined on $[a, b]$ and let $\phi$ be a convex function defined at least on the set $[m, M]$ where $m$ is the integral of $f$ and $M$ is the upper bound of $f$. Then,

$$
\phi\left(\frac{1}{b-a} \int_{a}^{b} f(x) d x\right) \leq \frac{1}{b-a} \int_{a}^{b} \phi(f(x) d x)
$$

By applying the Jensen's inequality with $\phi(y)=y^{2}, f(x)=q(t)$, and $t \in\left[t_{0}, t_{f}\right]\left(t_{f}-t_{0}=T\right)$, the resulting equation is equivalent to Equation (43), less than it is not a strict inequality:

$$
\left(\frac{1}{T} \int_{t_{0}}^{t_{f}} q_{j}(t) d t\right)^{2} \leq \frac{1}{T} \int_{t_{0}}^{t_{f}} q_{j}^{2}(t) d t
$$

However, the equality of Equation (45) holds only if $q_{j}(t)$ is a constant function, which contrasts with our assumption of analyzing the system in motion. Therefore, this is a strict inequality and both the conditions in Equations (41) and (42) are satisfied. The stationary point found in this case is a design of minimum energy for the system, corresponding to the global minimum of the unconstrained energy consumption function (Equation (13)). This design reduces the energy consumption of motor $j$ of the quantity $E_{s_{j}}$ :

$$
E_{j}=E_{w s_{j}}-\underbrace{\frac{a_{j}}{\mathrm{~K}_{r, j}} \cdot \frac{I_{\tau_{j}}^{2} I_{q_{j}^{2}}-2 I_{\tau_{j}} I_{q_{j}} I_{\tau q_{j}}+T I_{\tau q_{j}}^{2}}{I_{q_{j}}^{2}-T I_{q_{j}^{2}}}}_{E_{s_{j}}}
$$




\subsection{Active Constraint $\left(\mu^{*}>0\right)$}

When the design of minimum energy consumption lies outside the feasible region, the inequality is active and therefore binds the solution to the constraint:

$$
\begin{aligned}
\boldsymbol{\theta}_{j}^{*(2)} & =\frac{I_{\tau_{j}}}{T \mathrm{~K}_{r, j} k_{j}^{L}}+\frac{I_{q_{j}}}{T} \\
k_{j}^{*(2)} & =k_{j}^{L} \\
\mu^{*(2)} & =\frac{2 a_{j}}{T}\left(\mathrm{~K}_{r, j}\left(T I_{q_{j}^{2}}-I_{q_{j}}^{2}\right) k_{j}^{L}+T I_{\tau q_{j}}-I_{\tau_{j}} I_{q_{j}}\right)
\end{aligned}
$$

In order to verify that the solution found is a minimum, the Hessian of the Lagrangian function at the stationary point is evaluated:

$$
\left.\mathbf{H}=\nabla_{\mathbf{x}_{j} \mathbf{x}_{j}}^{2} \mathcal{L}\left(\mathbf{x}_{j}^{*(2)}, \mu_{j}^{*(2)}\right)=\left[\begin{array}{cc}
2 a_{j} T \mathrm{~K}_{r, j} k_{j}^{L^{2}} & 2 a_{j} I_{\tau_{j}} \\
2 a_{j} I_{\tau_{j}} & 2 a_{j}\left(\frac{I_{\boldsymbol{\tau}_{j}}^{2}}{T \mathrm{~K}_{r, j} k_{j}^{L^{2}}}+\frac{\mathrm{K}_{r, j}}{T}\left(T I_{q_{j}^{2}}-I_{q_{j}}^{2}\right)\right.
\end{array}\right)\right]
$$

$\mathbf{H}$ is positive definite if the determinant of all the upper-left sub-matrices are positive, i.e., if the following conditions hold:

$$
\begin{array}{cc}
\text { 1st condition: } & \operatorname{det}\left(\mathbf{H}_{11}\right)=2 a_{j} T \mathrm{~K}_{r, j} k_{j}^{L^{2}}>0 \\
\text { 2nd condition: } & \operatorname{det}(\mathbf{H})=\left(2 a_{j} \mathrm{~K}_{r, j} k_{j}^{L}\right)^{2}\left(T I_{q_{j}^{2}}-I_{q_{j}}^{2}\right)>0
\end{array}
$$

The first condition in Equation (51) is always satisfied as all the coefficients are positive. The second condition in Equation (52) is satisfied as the first factor is squared and the second one is always positive as demonstrated in Section 3.1. Therefore, the solution in Equations (47)-(49) is a minimum point of the Lagrangian function.

Determination of the Lower Bounds $k_{j}^{L}$

Although the solution outlined in Section 3.2 is a minimum of the bounded objective function, this does not ensure a reduction of the energy consumption with respect to the case without springs, as it does not correspond to the global minimum of the unconstrained energy consumption function (Equation (13)). In order for the addition of the springs to also be advantageous in this case, the value of the stiffness bound $k_{j}^{L}$ must be properly set. Let us evaluate the energy consumption when the design variables take the values $\boldsymbol{\theta}_{j}^{*(2)}$ and $k_{j}^{*(2)}$ (Equations (47) and (48)):

$$
E_{j}\left(\mathbf{x}_{j}^{*(2)}\right)=E_{w s_{j}}+\underbrace{\frac{a_{j}}{T \mathrm{~K}_{r, j}}\left(\mathrm{~K}_{r, j}^{2}\left(T I_{q_{j}^{2}}-I_{q_{j}}^{2}\right) k_{k}^{L^{2}}+2 \mathrm{~K}_{r, j}\left(T I_{\tau q_{j}}-I_{\tau_{j}} I_{q_{j}}\right) k_{j}^{L}-I_{\tau_{j}}^{2}\right)}_{E_{s_{j}}}
$$

In Equation (53), $E_{w s_{j}}$ is the energy consumption of the system without springs; therefore, in order to reduce the energy consumption $E_{j}$, the term $E_{s_{j}}$ must be negative. By solving for $k_{j}^{L}$ the inequality $E_{s_{j}}<0$ it is possible to find the range of values of the stiffness bounds allowing for energy reduction:

$$
0<k_{j}^{L}<\frac{I_{\tau_{j}} I_{q_{j}}-T I_{\tau q_{j}}+\sqrt{\left(T I_{\tau q_{j}}-I_{\tau_{j}} I_{q_{j}}\right)^{2}+I_{\tau_{j}}^{2}\left(T I_{q_{j}^{2}}-I_{q_{j}}^{2}\right)}}{\mathrm{K}_{r, j}\left(T I_{q_{j}^{2}}-I_{q_{j}}^{2}\right)}
$$


The selection of the most suitable value of $k_{j}^{L}$ inside the range in Equation (54) is to be chosen by the user depending on the specific system analyzed. As general guidelines, such selection is based on a trade-off between energy reduction and technical feasibility of the solution. For example, very small values of $k_{j}^{L}\left(k_{j}^{L} \rightarrow 0\right)$ lead to very large values for the mounting angle $\left(\theta_{j}^{*(2)} \rightarrow \infty\right.$, see Equation (47)), which may be physically impossible.

\section{Results}

Two test cases have been employed for the numerical validation of the proposed method: a five-bar mechanism and a SCARA robot. They are discussed in the following subsections.

\subsection{Five-Bar Mechanism}

The five-bar mechanism considered is shown in Figure 2a, while its dynamic properties are listed in Table 1. In this first test case the static and the viscous friction forces have been neglected $\left(\mathbf{f}_{s}=\mathbf{0}\right.$, $\left.\mathbf{F}_{v}=\mathbf{0}\right)$ as well as the interaction forces between the end-effector and the environment $\left(\mathbf{f}_{e}=\mathbf{0}\right)$, while both the cases in which the mechanism works on the horizontal $\left(\mathbf{f}_{g}=\mathbf{0}\right)$ or the vertical $\left(\mathbf{f}_{g} \neq \mathbf{0}\right)$ plane have been considered. The end-effector moves a payload of $2 \mathrm{~kg}$ following a typical pick and place trajectory, shown in Figure $2 b$, consisting in a vertical motion of $0.1 \mathrm{~m}$ between the Cartesian positions $\mathbf{P}_{0}=[-0.4,-0.6] \mathrm{m}$ and $\mathbf{P}_{1}=[-0.4,-0.5] \mathrm{m}$, a horizontal motion of $0.5 \mathrm{~m}$ from $\mathbf{P}_{1}$ to $\mathbf{P}_{2}=[0.1,-0.5]$ $\mathrm{m}$ and another vertical motion of $0.1 \mathrm{~m}$ from $\mathbf{P}_{2}$ to $\mathbf{P}_{3}=[0.1,-0.6] \mathrm{m}$.

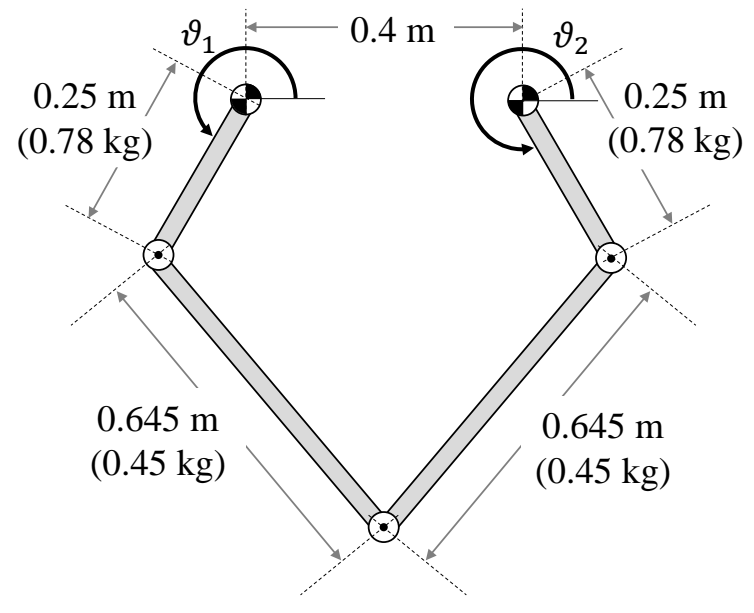

(a)

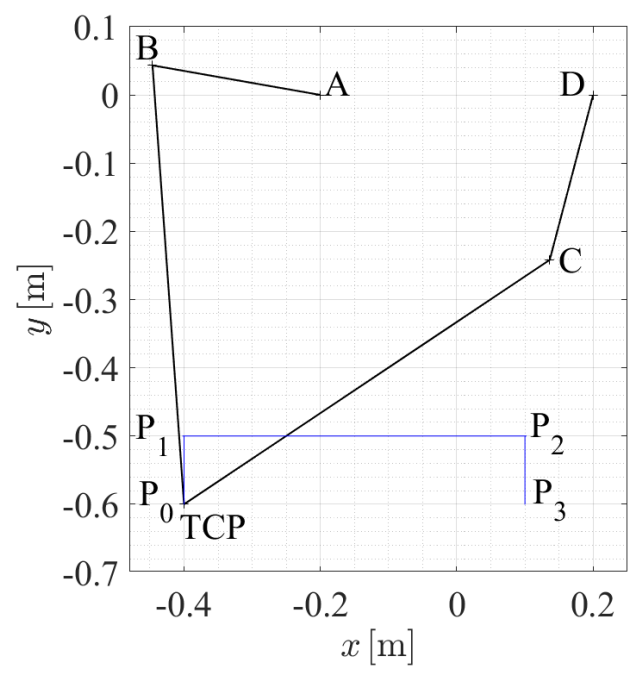

(b)

Figure 2. Five-bar mechanism: (a) kinematic schematic and (b) end-effector trajectory path in Cartesian space.

Table 1. Five-bar mechanism parameters.

\begin{tabular}{ccc}
\hline & Parameters & Joint 1 and Joint 2 \\
\hline $\mathrm{K}_{r}$ & Gear ratio $[-]$ & $1 / 20$ \\
$\mathrm{I}$ & Motor inertia $\left[\mathrm{kg} \cdot \mathrm{m}^{2}\right]$ & $4.13 \cdot 10^{-5}$ \\
$\mathrm{R}$ & Motor winding resistance $[\Omega]$ & 2.6 \\
$\mathrm{~K}_{e}$ & Motor velocity constant $[\mathrm{Vs} / \mathrm{rad}]$ & 0.42 \\
$\mathrm{~K}_{t}$ & Motor torque constant $[\mathrm{Nm} / \mathrm{A}]$ & 0.42 \\
$\eta$ & Efficiency $[-]$ & 1 \\
\hline
\end{tabular}

In such a task, the tool center point (TCP) moves from $\mathbf{P}_{0}$ to $\mathbf{P}_{3}$ and returns back in $T=1.6 \mathrm{~s}$. Each linear segment of the trajectory is executed following a double-S speed profile in the workspace 
with acceleration and deceleration times equal to $1 / 3 T_{i}$ and acceleration ramp rise and fall times equal to $1 / 10 T_{i}$, with $T_{i}$ the time period of each trajectory segment (see Figure $3 a$ ). The position, velocity and acceleration profiles of actuated joints corresponding to the chosen end-effector path have been computed by means of the inverse kinematics and are shown in Figure $3 b$.
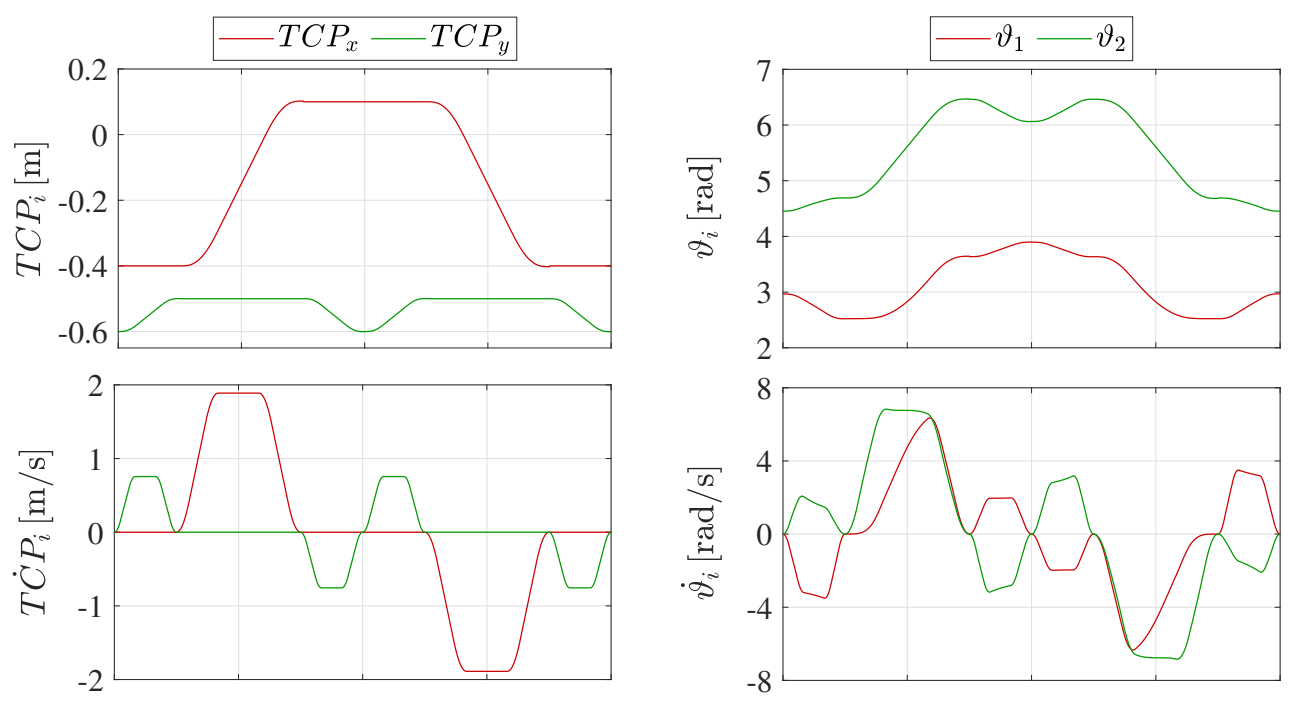

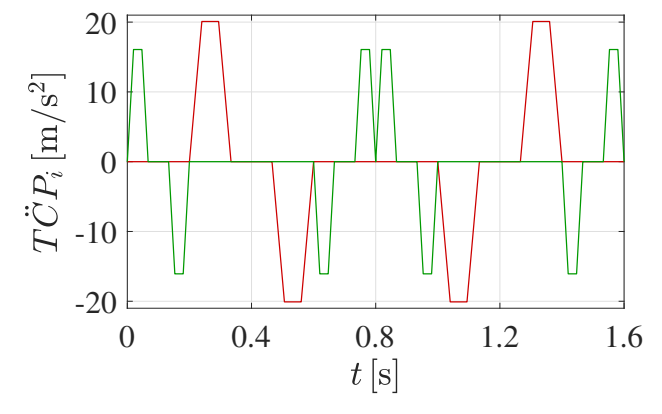

(a)

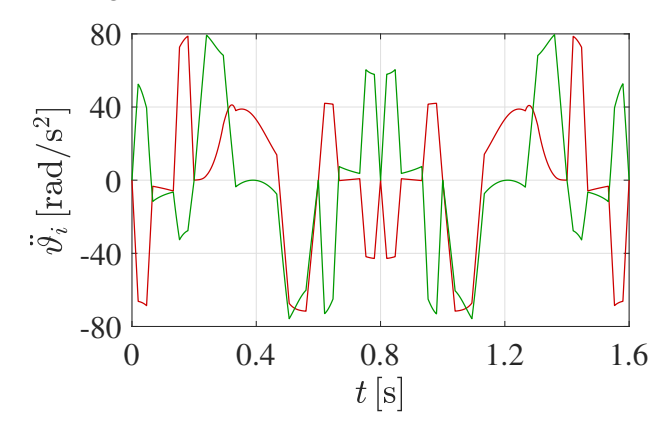

(b)

Figure 3. Five-bar mechanism trajectory planning: (a) TCP coordinates vs. time and (b) joint coordinates vs. time.

Both the motors of the system are equipped with regenerative drives and torsional springs designed following the approach described in Section 3. According to Equation (54), the lower bounds for the two springs must be included in the following range:

$$
\begin{array}{cl}
\text { vertical plane : } & \left\{\begin{array}{l}
0<k_{1}^{L}<17.23 \mathrm{Nm} / \mathrm{rad} \\
0<k_{2}^{L}<6.42 \mathrm{Nm} / \mathrm{rad}
\end{array}\right. \\
\text { horizontal plane : } & \left\{\begin{array}{l}
0<k_{1}^{L}<11.62 \mathrm{Nm} / \mathrm{rad} \\
0<k_{2}^{L}<8.92 \mathrm{Nm} / \mathrm{rad}
\end{array}\right.
\end{array}
$$

A lower bound equal to $1 \mathrm{Nm}$ has been set for both the spring stiffnesses in both the considered cases, as such a choice gives reasonable values for the variable $\boldsymbol{\theta}$ computed according to Equation (47):

$$
\begin{array}{cl}
\text { vertical plane : } & \left\{\begin{array}{l}
\boldsymbol{\theta}_{1}\left(k_{1}^{L}=1\right)=9.36 \mathrm{rad} \\
\boldsymbol{\theta}_{2}\left(k_{2}^{L}=1\right)=-3.09 \mathrm{rad}
\end{array}\right. \\
\text { horizontal plane : } & \left\{\begin{array}{l}
\boldsymbol{\theta}_{1}\left(k_{1}^{L}=1\right)=2.47 \mathrm{rad} \\
\boldsymbol{\theta}_{2}\left(k_{2}^{L}=1\right)=6.35 \mathrm{rad}
\end{array}\right.
\end{array}
$$


By solving the bounded optimization problem, the following optima are found:

$$
\begin{array}{cl}
\text { vertical plane : } & \begin{cases}\boldsymbol{\theta}_{1}^{*}=1.67 \mathrm{rad} & k_{1}^{*}=4.3 \mathrm{Nm} / \mathrm{rad} \\
\boldsymbol{\theta}_{2}^{*}=8.73 \mathrm{rad} & k_{2}^{*}=1.2 \mathrm{Nm} / \mathrm{rad} \\
\mu^{*}=0\end{cases} \\
\text { horizontal plane : } & \begin{cases}\boldsymbol{\theta}_{1}^{*}=3.00 \mathrm{rad} & k_{1}^{*}=5.7 \mathrm{Nm} / \mathrm{rad} \\
\boldsymbol{\theta}_{2}^{*}=5.70 \mathrm{rad} & k_{2}^{*}=4.4 \mathrm{Nm} / \mathrm{rad} \\
\mu^{*}=0\end{cases}
\end{array}
$$

In both cases, the solution found lies inside the feasible region of the constraint and corresponds to the minimum of the unconstrained objective function. The time histories of the electric power required by the two motors provided with the optimal springs are shown in the second row of Figures 4 and 5 for the case with and without gravity, respectively. The same figures also show in the first row the electric power required by the system without torsional springs. The comparison between the areas delimited by the electric power curves when springs are used or not clearly shows that the springs reduces the energy consumption in all the scenarios considered.
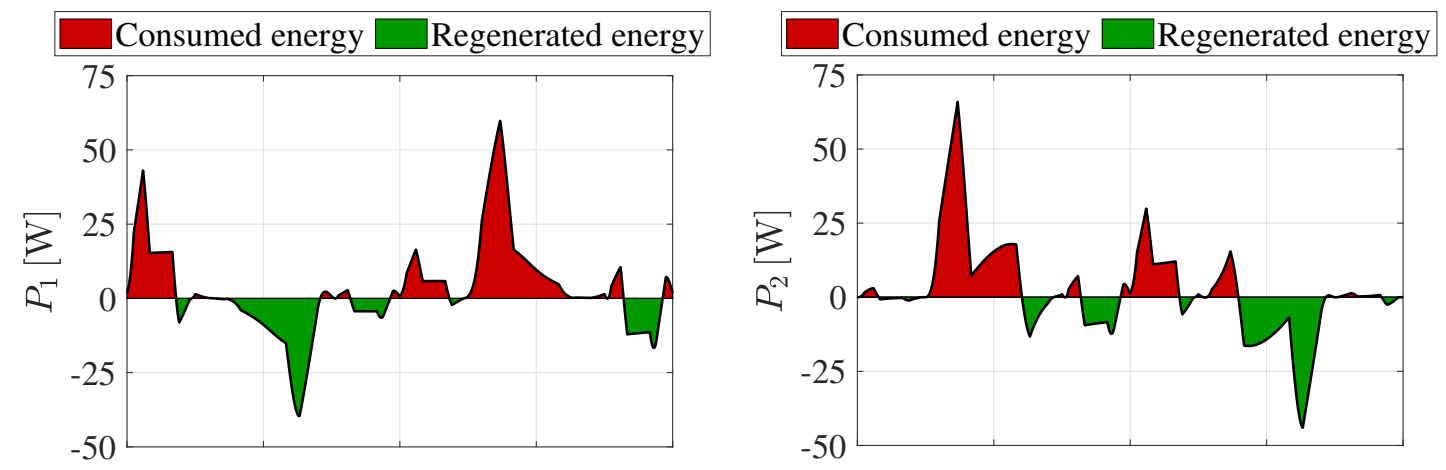

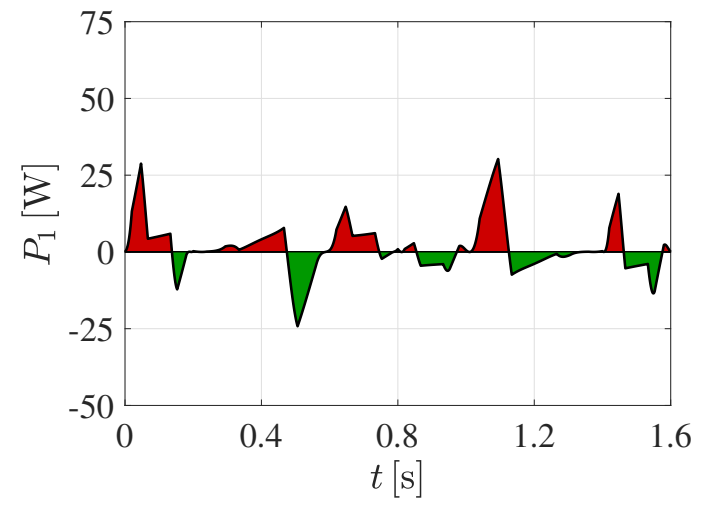

(a)

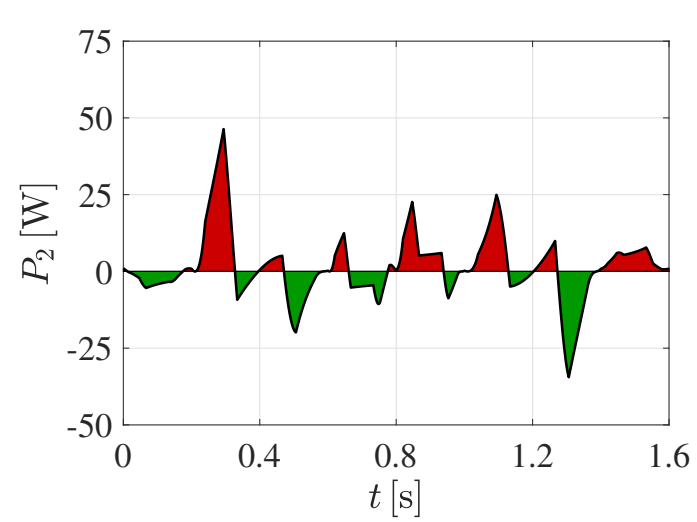

(b)

Figure 4. Electric power without (top) and with (bottom) springs of motor 1 (a) and motor 2 (b) when the mechanism lies on the vertical plane.

In order to corroborate the effectiveness of the co-exploitation of either energy-recovering devices and elastic-energy storing systems, in Table 2 the values of the consumed energy per cycle for the original system and the one retrofitted following the proposed approach are reported.

The values of the energy reduction clearly demonstrate that a great saving can be achieved with the suggested retrofit of the system. By comparing the consumed energy values obtained considering or not the effects of the gravity forces (i.e., vertical or horizontal plane), it is evident that the spring torques do not compensate only the self-weight of the manipulator. Their use appears really advantageous 
also with no-gravity to compensate the inertial forces, leading to an overall reduction of the energy consumption of about $70 \%$.
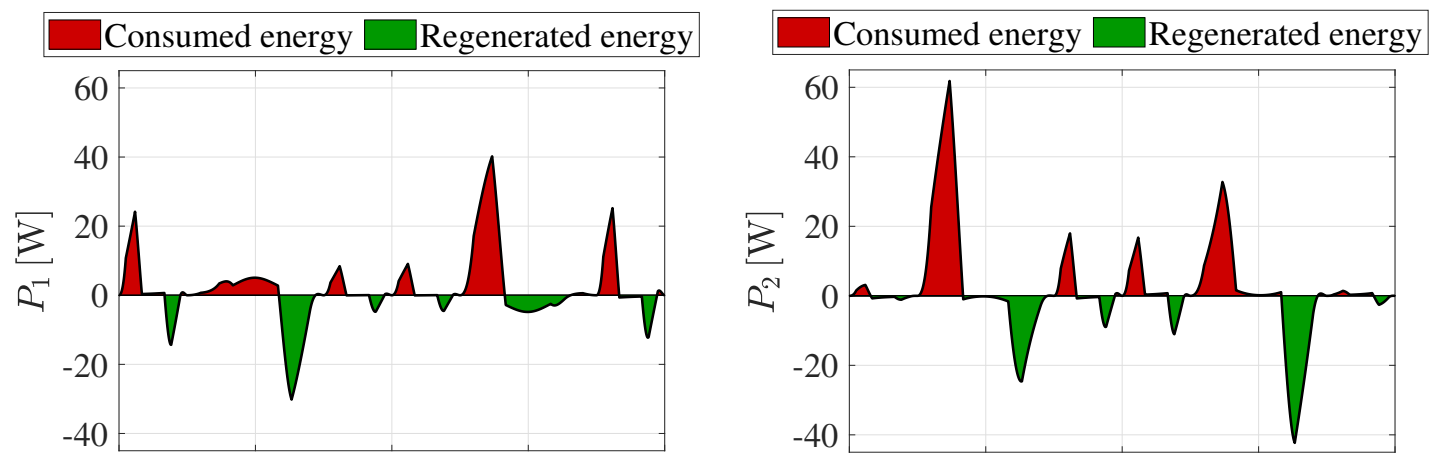

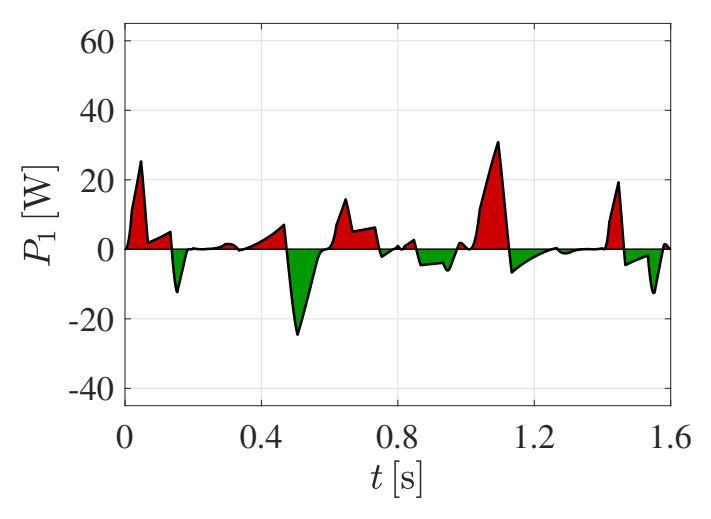

(a)

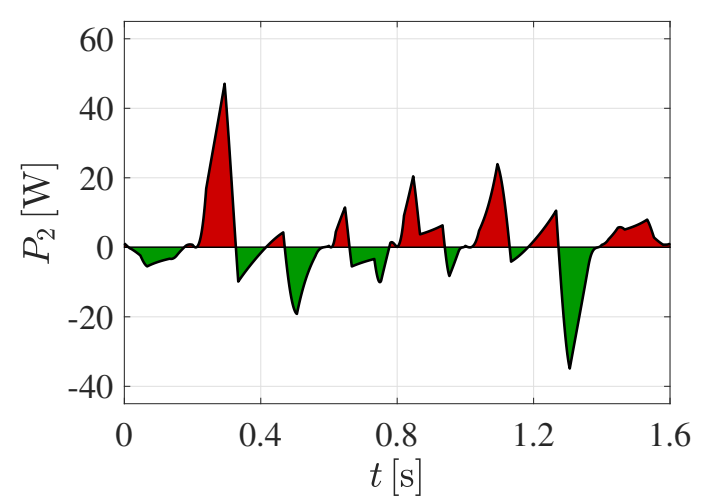

(b)

Figure 5. Electric power without (top) and with (bottom) springs of motor 1 (a) and motor 2 (b) when the mechanism lies on the horizontal plane.

Table 2. Consumed energy per cycle with $(\bullet)$ or without (०) energy reducing devices.

\begin{tabular}{ccccccc}
\hline \multirow{3}{*}{ Motor } & \multicolumn{3}{c}{ Vertical Plane } & \multicolumn{3}{c}{ Horizontal Plane } \\
& $\begin{array}{c}\text { Energy (J) } \\
\text { Energy }\end{array}$ & $\begin{array}{c}\text { Energy } \\
\text { Reduction (\%) }\end{array}$ & $\circ$ & $\bullet$ & $\begin{array}{c}\text { Energy (J) } \\
\text { Reduction (\%) }\end{array}$ \\
\hline 1 & 10.15 & 2.06 & 79.70 & 5.62 & 1.88 & 66.59 \\
2 & 9.43 & 2.29 & 75.69 & 7.31 & 2.14 & 70.63 \\
Total & 19.58 & 4.35 & 77.78 & 12.93 & 4.02 & 68.90 \\
\hline
\end{tabular}

\subsection{SCARA Robot}

As the second test case a 4-dof SCARA robot is used. The kinematic diagram scheme and the geometrical dimensions of the robot are shown in Figure 6a, while its main electric and mechanical parameters are reported in Table 3. The system performs a linear rest to rest motion of the end-effector between the Cartesian positions $\mathbf{P}_{0}=[-0.175,0.400,0.300] \mathrm{m}$ and $\mathbf{P}_{1}=[0.175,0.350,0.200] \mathrm{m}$ with initial and final orientation angles about the z-axis of $\pi / 3 \mathrm{rad}$ and $\pi \mathrm{rad}$, respectively (see Figure $6 \mathrm{~b}$ ), and returns back to $\mathbf{P}_{0}$. 


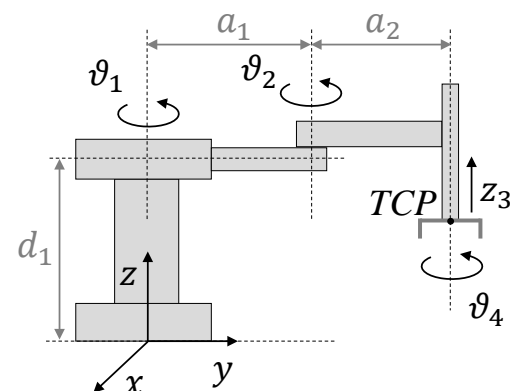

Denavit-Hartenberg parameters

\begin{tabular}{|c|c|c|c|c|}
\hline$i$ & $\begin{array}{c}\alpha_{i} \\
{[\mathrm{rad}]}\end{array}$ & $\begin{array}{c}a_{i} \\
{[\mathrm{~mm}]}\end{array}$ & $\begin{array}{c}d_{i} \\
{[\mathrm{~mm}]}\end{array}$ & $\begin{array}{c}\vartheta_{i} \\
{[\mathrm{rad}]}\end{array}$ \\
\hline 1 & 0 & 425 & 398 & $\vartheta_{1}$ \\
\hline 2 & 0 & 375 & 0 & $\vartheta_{2}$ \\
\hline 3 & 0 & 0 & $z_{3}$ & 0 \\
\hline 4 & 0 & 0 & 0 & $\vartheta_{3}$ \\
\hline
\end{tabular}

(a)

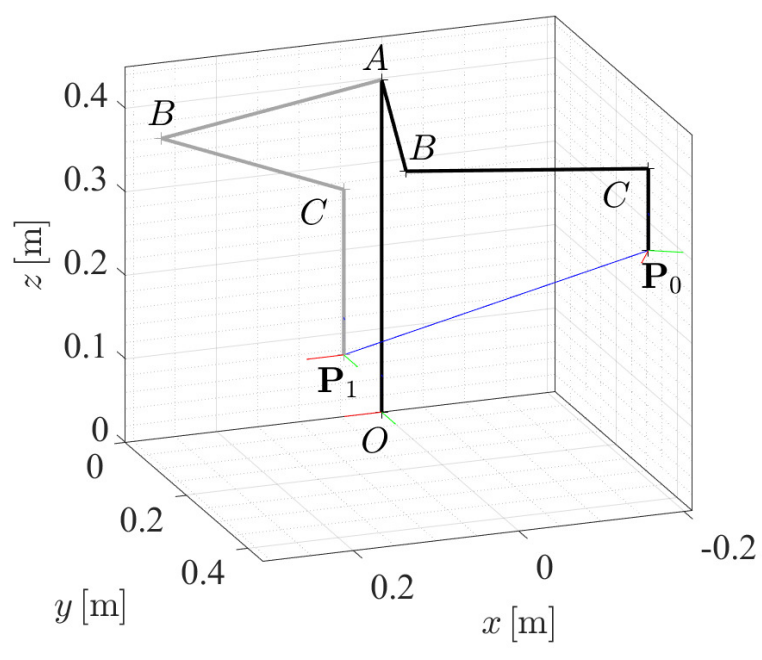

(b)

Figure 6. SCARA robot: (a) kinematic schematic and (b) end-effector trajectory path in Cartesian space.

Table 3. SCARA parameters.

\begin{tabular}{ccccc}
\hline Parameters & Joint 1 & Joint 2 & Joint 3 & Joint 4 \\
\hline $\mathrm{M}[\mathrm{kg}]$ & 12 & 15 & - & 2 \\
$\mathrm{~K}_{r}[-, \mathrm{m} / \mathrm{rad}]$ & $1 / 60$ & $1 / 100$ & $1 / 210$ & $1 / 30$ \\
$\mathrm{I}\left[\mathrm{kgm}^{2}\right]$ & $2.08 \cdot 10^{-5}$ & $2.08 \cdot 10^{-5}$ & $1.70 \cdot 10^{-6}$ & $1.70 \cdot 10^{-6}$ \\
$\mathrm{~F}_{v}[\mathrm{Nms} / \mathrm{rad}, \mathrm{Ns} / \mathrm{m}]$ & 0.03 & 0.03 & 0.2 & 0.001 \\
$\mathrm{~F}_{S}[\mathrm{Nm}, \mathrm{N}]$ & 0.4 & 0.4 & 0.1 & 0.02 \\
$\mathrm{R}[\Omega]$ & 39.4 & 39.4 & 18.2 & 18.2 \\
$\mathrm{~K}_{e}[\mathrm{Vs} / \mathrm{rad}]$ & 0.588 & 0.588 & 0.16 & 0.16 \\
$\mathrm{~K}_{t}[\mathrm{Nm} / \mathrm{A}]$ & 0.588 & 0.588 & 0.16 & 0.16 \\
$\boldsymbol{\eta}[-]$ & 1 & 1 & 1 & 1 \\
\hline
\end{tabular}

Fifth-degree polynomial profiles have been chosen for the motion planning in the operative space as shown in Figure 7. The same figure also shows the corresponding position, velocity, and acceleration profiles of actuated joints. For this second test case, it has been assumed to provide all the four motors with regenerative drives. Conversely, the elastic elements are added only to the first two joints, that are the ones undergoing the largest motion. A lower bound of $5 \mathrm{Nm}$ has been set for both the stiffnesses $\left(k_{1}^{L}, k_{2}^{L}\right)$ which is inside their admissible ranges:

$$
\begin{aligned}
& 0<k_{1}^{L}<84.5 \mathrm{Nm} / \mathrm{rad} \\
& 0<k_{2}^{L}<180 \mathrm{Nm} / \mathrm{rad}
\end{aligned}
$$

For the analyzed trajectory the optimum spring design is $k_{1}^{*}=42 \mathrm{Nm} / \mathrm{rad}, \boldsymbol{\theta}_{1}^{*}=0.7418 \mathrm{rad}$, $k_{2}^{*}=59 \mathrm{Nm} / \mathrm{rad}$, and $\boldsymbol{\theta}_{2}^{*}=1.88 \mathrm{rad}$. Such a design leads to a reduction of the electric energy consumption as it can be seen by comparing the areas delimited by the electric power curves in Figure 8 when torsional springs in parallel with the motors are used (second row) or not (first row). 

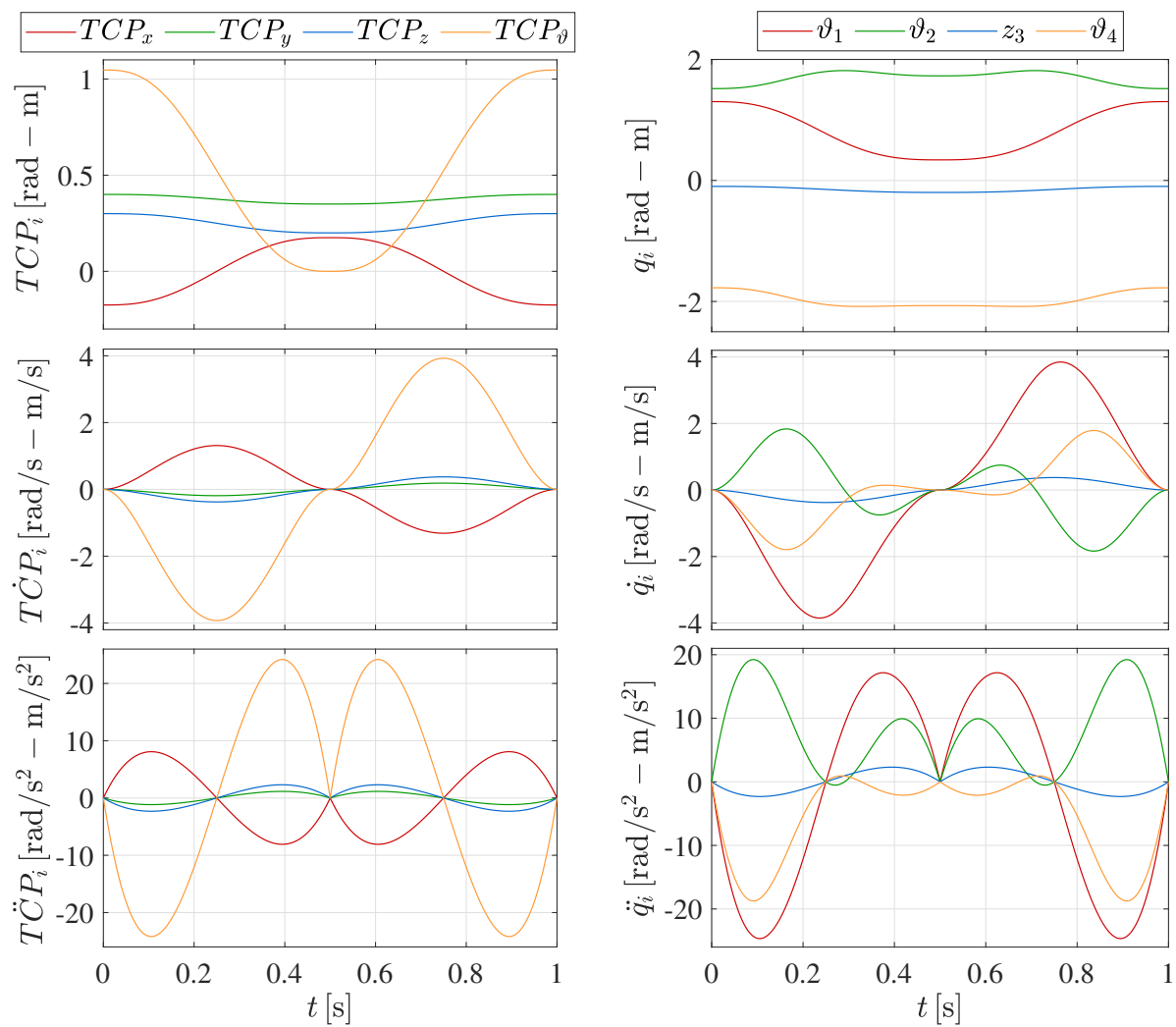

(a)

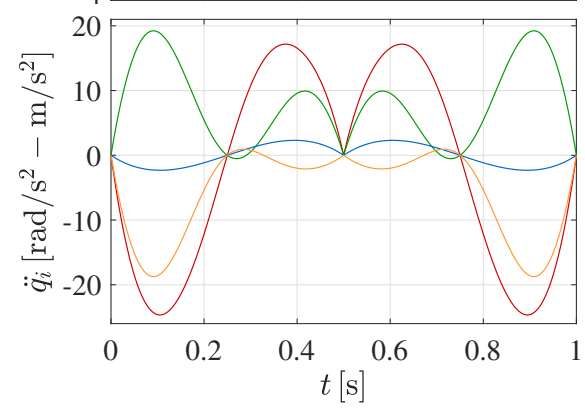

(b)

Figure 7. SCARA trajectory planning: (a) TCP coordinates vs. time and (b) joint coordinates vs time.

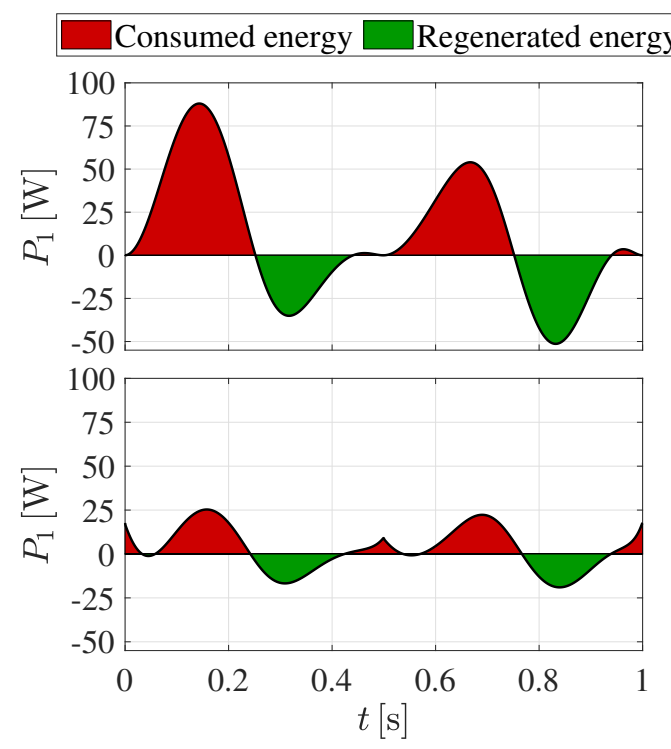

(a)
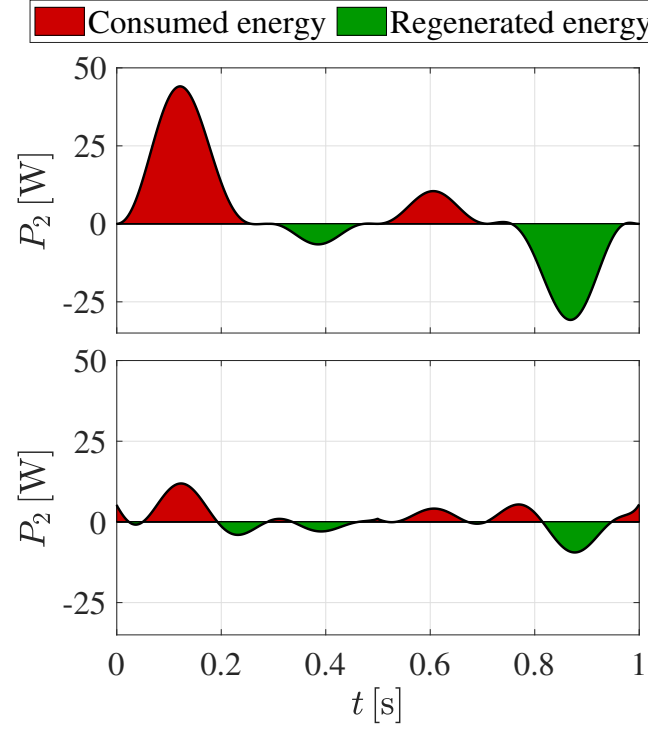

(b)

Figure 8. Electric power without (top) and with (bottom) springs of motor 1 (a) and motor 2 (b).

In order to quantify the benefits of the concurrent addition of regenerative drives and compliant elements to the system, Table 4 states the values of the consumed energy per cycle employing or not such devices. 
Table 4. Consumed energy per cycle employing $(\bullet)$ or not (०) energy reducing devices.

\begin{tabular}{cccc}
\hline Motor & \multicolumn{2}{c}{ Energy (J) } & $\begin{array}{c}\text { Energy } \\
\text { Reduction (\%) }\end{array}$ \\
\hline 1 & 19.69 & 2.50 & 87.28 \\
2 & 6.38 & 0.6689 & 89.53 \\
3 & 19.68 & 14.30 & 27.35 \\
4 & 1.97 & 1.50 & 24.03 \\
\hline Total & 47.72 & 18.96 & 60.26 \\
\hline
\end{tabular}

By comparing the consumed energy reduction of motors 3 and 4 with that of motors 1 and 2, it appears evident the positive contribution of the compliant elements in the minimization of the energy consumption. Indeed, motors 3 and 4 are provided only with regenerative drives and the energy reduction is on average about $25.5 \%$, while an energy saving up to $89 \%$ is obtained for motors 1 and 2 that exploit both energy reducing devices. Overall, a very satisfactory result is obtained being the system energy consumption reduced about $60 \%$.

\section{Conclusions}

This work has presented a method to minimize the energy consumption of robotic systems performing cyclic tasks. The method relies on a retrofit of the system by means of energy-recovering and energy-storing devices, such as regenerative drives and compliant elements. The first device allows to harvest instead of waste the system braking energy, while the second stores potential elastic energy. Both these sources of energy are then transferred to the system reducing the energy needed by the actuators.

In order to enhance the energy efficiency of the system, the compliant elements must be optimally designed in terms of stiffness and preload. To this end, a bounded optimization problem has been solved analytically. This allows the determination of the optimal compliant element parameters that minimize the motor electrical energy consumption for a given periodic path executed by the robot.

The method has been applied to a five-bar mechanism and to a four-dof SCARA robot. The results demonstrate how this technique has distinct advantages in terms of reduction of consumed energy that can reach values as high as $77.8 \%$ with a standard, not-optimized trajectory. Further trajectory optimization could lead to even further reduction.

Author Contributions: Conceptualization, I.P., E.W., G.C., and R.V.; methodology, I.P. and E.W.; software, I.P., E.W. and G.C.; validation, I.P.; formal analysis, I.P.; writing — original draft preparation, I.P.; writing - review and editing, E.W., G.C., and R.V.; supervision, R.V. All authors have read and agreed to the published version of the manuscript.

Funding: This research received no external funding.

Conflicts of Interest: The authors declare no conflict of interest.

\section{References}

1. Paryanto; Brossog, M.; Bornschlegl, M.; Franke, J. Reducing the energy consumption of industrial robots in manufacturing systems. Int. J. Adv. Manuf. Technol. 2015, 78, 1315-1328. [CrossRef]

2. Carabin, G.; Wehrle, E.; Vidoni, R. A review on energy-saving optimization methods for robotic and automatic systems. Robotics 2017, 6, 39. [CrossRef]

3. Scalera, L.; Boscariol, P.; Carabin, G.; Vidoni, R.; Gasparetto, A. Enhancing energy efficiency of a 4-DOF parallel robot through task-related analysis. Machines 2020, 8, 10. [CrossRef]

4. Ruiz, A.; Fontes, J.; Da Silva, M. The influence of kinematic redundancies in the energy efficiency of planar parallel manipulators. In Proceedings of the ASME International Mechanical Engineering Congress and Exposition (IMECE), Houston, TA, USA, 13-19 November 2015; Volume 4A-2015. [CrossRef]

5. Boscariol, P.; Richiedei, D. Trajectory design for energy savings in redundant robotic cells. Robotics 2019, 8, 15. [CrossRef] 
6. Boscariol, P.; Caracciolo, R.; Richiedei, D.; Trevisani, A. Energy optimization of functionally redundant robots through motion design. Appl. Sci. 2020, 10, 22. [CrossRef]

7. Carabin, G.; Vidoni, R.; Wehrle, E. Energy saving in mechatronic systems through optimal point-to-point trajectory generation via standard primitives. Mech. Mach. Sci. 2019, 68, 20-28. [CrossRef]

8. Boscariol, P.; Richiedei, D. Energy-efficient design of multipoint trajectories for Cartesian robots. Int. J. Adv. Manuf. Technol. 2019, 102, 1853-1870. [CrossRef]

9. Hansen, C.; Kotlarski, J.; Ortmaier, T. Optimal motion planning for energy efficient multi-axis applications. Int. J. Mechatronics Autom. 2014, 4, 147-160. [CrossRef]

10. Khalaf, P.; Richter, H. Parametric optimization of stored energy in robots with regenerative drive systems. In Proceedings of the 2016 IEEE International Conference on Advanced Intelligent Mechatronics (AIM), Banff, AB, Canada, 12-15 July 2016; pp. 1424-1429.

11. Kim, Y.J. Design of low inertia manipulator with high stiffness and strength using tension amplifying mechanisms. In Proceedings of the IEEE International Conference on Intelligent Robots and Systems, Hamburg, Germany, 28 September-2 October 2015; Volume 2015-December, pp. 5850-5856. [CrossRef]

12. Yin, H.; Liu, J.; Yang, F. Hybrid Structure Design of Lightweight Robotic Arms Based on Carbon Fiber Reinforced Plastic and Aluminum Alloy. IEEE Access 2019, 7, 64932-64945. [CrossRef]

13. Meike, D.; Ribickis, L. Recuperated energy savings potential and approaches in industrial robotics. In Proceedings of the 2011 IEEE International Conference on Automation Science and Engineering, Trieste, Italy, 24-27 August 2011; pp. 299-303.

14. Sayed-Ahmed, A.; Wei, L.; Seibel, B. Industrial regenerative motor-drive systems. In Proceedings of the 2012 Twenty-Seventh Annual IEEE Applied Power Electronics Conference and Exposition (APEC), Orlando, FL, USA, 5-9 Febuary 2012; pp. 1555-1561.

15. Kapoor, R.; Parveen, C. Comparative study on various KERS. In Proceedings of the Lecture Notes in Engineering and Computer Science, London, UK, 3-5 July 2013; Volume 3 LNECS, pp. 1969-1973.

16. Rankis, I.; Meike, D.; Senfelds, A. Utilization of Regeneration Energy in Industrial Robots System. In Proceedings of the International Conference on Renewable Energy and Environmental Technology (REET 2013), Jilin, China, 21-22 September 2013; pp. 448-453.

17. Gale, S.; Eielsen, A.; Gravdahl, J. Modelling and simulation of a flywheel based energy storage system for an industrial manipulator. In Proceedings of the IEEE International Conference on Industrial Technology, Seville, Spain, 17-19 March 2015; Volume 2015-June, pp. 332-337. [CrossRef]

18. Verstraten, T.; Beckerle, P.; Furnémont, R.; Mathijssen, G.; Vanderborght, B.; Lefeber, D. Series and parallel elastic actuation: Impact of natural dynamics on power and energy consumption. Mech. Mach. Theory 2016, 102, 232-246. [CrossRef]

19. Scalera, L.; Palomba, I.; Wehrle, E.; Gasparetto, A.; Vidoni, R. Natural motion for energy saving in robotic and mechatronic systems. Appl. Sci. 2019, 9, 3516. [CrossRef]

20. Veer, S.; Sujatha, S. Approximate spring balancing of linkages to reduce actuator requirements. Mech. Mach. Theory 2015, 86, 108-124. [CrossRef]

21. Martini, A.; Troncossi, M.; Rivola, A. Algorithm for the static balancing of serial and parallel mechanisms combining counterweights and springs: Generation, assessment and ranking of effective design variants. Mech. Mach. Theory 2019, 137, 336-354. [CrossRef]

22. Barreto, J.; Schöler, F.F.; Corves, B. The concept of natural motion for pick and place operations. Mech. Mach. Sci. 2017, 46, 89-98. [CrossRef]

23. Barreto, J.P.; Corves, B. Matching the Free-Vibration Response of a Delta Robot with Pick-and-Place Tasks Using Multi-Body Simulation. In Proceedings of the 2018 IEEE 14th International Conference on Automation Science and Engineering (CASE), Munich, Germany, 20-24 August 2018; pp. 1487-1492.

24. Kashiri, N.; Spyrakos-Papastavridis, E.; Caldwell, D.; Tsagarakis, N. Exploiting the natural dynamics of compliant joint robots for cyclic motions. In Proceedings of the 2017 22nd International Conference on Methods and Models in Automation and Robotics, (MMAR 2017), Miedzyzdroje, Poland, 28-31 August 2017; pp. 676-681. [CrossRef]

25. Schiehlen, W.; Iwamura, M. Minimum energy control of multibody systems utilizing storage elements. In Proceedings of the ASME 2009 International Design Engineering Technical Conferences and Computers and Information in Engineering Conference, San Diego, CA, USA, 30 August-2 September 2009; American Society of Mechanical Engineers: New York, NY, USA, 2009; pp. 1919-1926. 
26. Iwamura, M.; Imafuku, S.; Kawamoto, T.; Schiehlen, W. Design and Control of an Energy-Saving Robot Using Storage Elements and Reaction Wheels. In Multibody Dynamics; Springer: Cham, Switzerland, 2016; pp. 277-297.

27. Schmit, N.; Okada, M. Simultaneous optimization of robot trajectory and nonlinear springs to minimize actuator torque. In Proceedings of the 2012 IEEE International Conference on Robotics and Automation (ICRA), Saint Paul, MN, USA, 14-18 May 2012; pp. 2806-2811.

28. Bidgoly, H.J.; Parsa, A.; Yazdanpanah, M.J.; Ahmadabadi, M.N. Benefiting From Kinematic Redundancy Alongside Mono-and Biarticular Parallel Compliances for Energy Efficiency in Cyclic Tasks. IEEE Trans. Robot. 2017, 33, 1088-1102. [CrossRef]

29. Zhang, Q.; Zhao, M.Y. Minimum time path planning of robotic manipulator in drilling/spot welding tasks. J. Comput. Des. Eng. 2016, 3, 132-139. [CrossRef]

30. Rojas, R.; Garcia, M.; Wehrle, E.; Vidoni, R. A Variational Approach to Minimum-Jerk Trajectories for Psychological Safety in Collaborative Assembly Stations. IEEE Robot. Autom. Lett. 2019, 4, 823-829. [CrossRef]

31. Boscariol, P.; Boschetti, G.; Gallina, P.; Passarini, C. Spring design for motor torque reduction in articulated mechanisms. Mech. Mach. Sci. 2018, 49, 557-564. [CrossRef]

32. Scalera, L.; Carabin, G.; Vidoni, R.; Wongratanaphisan, T. Energy efficiency in a 4-dof parallel robot featuring compliant elements. Int. J. Mech. Control 2019, 20, 1-9.

33. Richiedei, D.; Trevisani, A. Optimization of the Energy Consumption Through Spring Balancing of Servo-Actuated Mechanisms. J. Mech. Des. 2019, 142. [CrossRef]

34. Carabin, G.; Palomba, I.; Wehrle, E.; Vidoni, R. Energy Expenditure Minimization for a Delta-2 Robot Through a Mixed Approach. Comput. Methods Appl. Sci. 2020, 53, 383-390.. [CrossRef]

35. Richiedei, D.; Trevisani, A. Analytical computation of the energy-efficient optimal planning in rest-to-rest motion of constant inertia systems. Mechatronics 2016, 39, 147-159. [CrossRef]

36. Karush, W. Minima of Functions of Several Variables with Inequalities as Side Constraints. Master's Thesis, Department of Mathematics, University of Chicago, Chicago, IL, USA, 1939.

37. Kuhn, H.W.; Tucker, A.W. Nonlinear Programming, Proceedings of the Second Berkeley Symposium on Mathematical Statistics and Probability, Department of Mathematics, University of California, CA, USA, 31 July-12 August 1950; University of California Press: Berkeley, CA, USA, 1951; pp. 481-492. [CrossRef]

Publisher's Note: MDPI stays neutral with regard to jurisdictional claims in published maps and institutional affiliations.

(C) 2020 by the authors. Licensee MDPI, Basel, Switzerland. This article is an open access article distributed under the terms and conditions of the Creative Commons Attribution (CC BY) license (http://creativecommons.org/licenses/by/4.0/). 